\begin{tabular}{|c|c|}
\hline Title & $\begin{array}{l}\text { A n epigenome-wide study of cord blood DNA methylations in relation to prenatal perfluoroal kyl substance exposure: } \\
\text { The Hokkaido study }\end{array}$ \\
\hline Author(s) & $\begin{array}{l}\text { Miura, Ryu; A raki, A tsuko; Miyashita, Chihiro; Kobayashi, Sumitaka; Kobayashi, Sachiko; W ang, Shu-Li; Chen, } \\
\text { Chung-Hsing; Miyake, Kunio; I shizuka, Mayumi; Iwasaki, Y usuke; Ito, Y oichi M.; Kubota, Takeo; Kishi, Reiko }\end{array}$ \\
\hline Citation & $\begin{array}{l}\text { Environment international, 115, 21-28 } \\
\text { https://doi.org/10.1016/.envint.2018.03.004 }\end{array}$ \\
\hline Issue Date & $2018-06$ \\
\hline Doc URL & http:/hdl .handle.net/2115/78252 \\
\hline Rights & $\begin{array}{l}\text { (c) 2018. This manuscript version is made available under the CC-BY-NC-ND } 4.0 \text { license } \\
\text { http://creativecommons.org/icenses/by-nc-nd/4.0/ }\end{array}$ \\
\hline Rights(URL) & http://creativecommons.org/icenses/by-nc-nd/4.0/ \\
\hline Tyре & article (author version) \\
\hline Additional Information & There are other files related to this item in HUSCAP. Check the above URL. \\
\hline File Information & Environlnt115_21.pdf \\
\hline
\end{tabular}

Instructions for use 


\section{An epigenome-wide study of cord blood DNA methylations in relation to prenatal}

\section{perfluoroalkyl substance exposure: the Hokkaido study}

Ryu Miura ${ }^{1}$, Atsuko Araki ${ }^{1}$, Chihiro Miyashita ${ }^{1}$, Sumitaka Kobayashi ${ }^{1}$, Sachiko

Kobayashi $^{1}$, Shu-Li Wang ${ }^{2}$, Chung-Hsing Chen ${ }^{3,4}$, Kunio Miyake ${ }^{5}$, Mayumi Ishizuka ${ }^{6}$,

Yusuke Iwasaki ${ }^{7}$, Yoichi M. Ito ${ }^{8}$, Takeo Kubota ${ }^{9}$, Reiko Kishi $^{1}$

${ }^{1}$ Hokkaido University Center for Environmental and Health Sciences, Sapporo, Japan

${ }^{2}$ National Institute of Environmental Health Sciences, National Health Research

Institutes, Zhunan, Taiwan

${ }^{3}$ National Institute of Cancer Research, National Health Research Institutes, Zhunan,

Taiwan

${ }^{4}$ Taiwan Bioinformatics Core, National Health Research Institutes, Zhunan, Taiwan

${ }^{5}$ Department of Health Sciences, Interdisciplinary Graduate School of Medicine and

Engineering, University of Yamanashi, Japan

${ }^{6}$ Department of Environmental Veterinary Sciences, Graduate School of Veterinary

Medicine, Hokkaido University, Sapporo, Japan

${ }^{7}$ Department of Physiology and Molecular Sciences, Hoshi University, Tokyo, Japan

${ }^{8}$ Department of Biostatistics, Graduate School of Medicine, Hokkaido University,

Sapporo, Japan 
${ }^{9}$ Faculty of Child Studies, Seitoku University, Chiba, Japan.

Correspondence: Reiko Kishi, MD, PhD, MPH, Eminent Professor, Center for

Environmental and Health Sciences, Hokkaido University, North 12 West 7, Kita-ku,

Sapporo, 060-0812, Japan. Tel.: +81-11-706-4746. Fax: +81-11-706-4725. E-mail:

rkishi@med.hokudai.ac.jp

Disclosure statement: The authors have no conflicts of interest to disclose. 


\begin{abstract}
Background: Prenatal exposure to perfluoroalkyl substances (PFASs) influences fetal development and later in life.
\end{abstract}

Objective: To investigate cord blood DNA methylation changes associated with prenatal exposure to PFASs.

Methods: We assessed DNA methylation in cord blood samples from 190 mother-child pairs from the Sapporo cohort of the Hokkaido Study (discovery cohort) and from 37 mother-child pairs from the Taiwan Maternal and Infant Cohort Study (replication cohort) using the Illumina HumanMethylation 450 BeadChip. We examined the associations between methylation and PFAS levels in maternal serum using robust linear regression models and identified differentially methylated positions (DMPs) and regions (DMRs).

Results: We found four DMPs with a false discovery rate below 0.05 in the discovery cohort. Among the top 20 DMPs ranked by the lowest $P$-values for perfluorooctane sulfonate (PFOS) and perfluorooctanoic acid (PFOA) exposure, four DMPs showed the same direction of effect and $P$-value $<0.05$ in the replication assay: cg16242615 mapped to ZBTB7A, cg21876869 located in the intergenic region (IGR) of USP2-AS1, cg00173435 mapped to TCP11L2, and cg18901140 located in IGR of NTN1. For DMRs, we found a region associated with PFOA exposure with family-wise error rate $<0.1$ 
located in ZFP57, showing the same direction of effect in the replication cohort. Among the top five DMRs ranked by the lowest $P$-values that were associated with exposure to PFOS and PFOA, in addition to ZFP57, DMRs in the CYP2E1, SMAD3, SLC17A9, GFPT2, DUSP22, and TCERG1L genes showed the same direction of effect in the replication cohort.

Conclusion: We suggest that prenatal exposure to PFASs may affect DNA methylation status at birth. Longitudinal studies are needed to examine whether methylation changes observed are associated with differential health outcomes.

Key words: DNA methylation; perfluoroalkyl substances; prenatal exposure; genome-wide association; cord blood

Abbreviations:

PFASs, perfluoroalkyl substances

DM, differentially methylated

DMP, DM position

DMR, DM region

CpG, cytosine-guanine dinucleotide 
PFOS, perfluorooctane sulfonate

PFOA, perfluorooctanoic acid

Coef, partial regression coefficient

FDR, false discovery rate

FWER, family-wise error rate

LC-MS/MS, column-switching liquid chromatography-tandem mass spectrometry

TMICS, Taiwan Maternal and Infant Cohort Study

KEGG, Kyoto Encyclopedia Genes and Genomes

IGR, intergenic region

TSS200, the region of 200 bp upstream of the transcription start site

TSS1500, the region of 1500 bp upstream of the transcription start site 


\section{Introduction}

During the prenatal period, the fetus undergoes dynamic changes to develop its body from a single fertilized egg in under ten months. Any perturbations of this process by external factors may confer abnormal phenotypes and affect future health trajectories of the offspring. Perfluoroalkyl substances (PFASs) are synthetic compounds ubiquitously distributed in the environment that can disrupt endocrine system functions (Lau et al. 2007). PFASs have long half-lives in human: 5.4 years for perfluorooctane sulfonate (PFOS) and 3.8 years for perfluorooctanoic acid (PFOA) (Olsen et al. 2007). Furthermore, PFASs can pass through the placental barrier (Inoue et al. 2004). Consequently, fetuses can be exposed to PFASs via maternal circulation, which suggests a possibility of PFAS negative effects on embryonic and fetal development.

Experimental studies have shown associations of exposures to PFASs with developmental and reproductive toxicity (Abbott et al. 2007; Luebker et al. 2005) as well as with immunotoxicity (DeWitt et al. 2012). It has also been revealed that PFASs disturb metabolic end-points, including lipid metabolism, glucose homeostasis, and thyroid hormone balance (Seacat et al. 2003; Thibodeaux et al. 2003), and neuronal physiological processes in the developing brain (Johansson et al. 2008, 2009; Liao et al. 
2008, 2009). Moreover, epidemiological studies have shown that prenatal exposure to PFASs has been associated with various health outcomes, including birth size reduction, disruption of hormone balance, obesity, neurodevelopmental problems, and immune function impairment (Apelberg et al. 2007; Chen et al. 2013; Grandjean et al. 2012; Halldorsson et al. 2012; Kishi et al. 2017; Olsen et al. 2009). However, the mechanisms underlying these associations are not clear. One hypothesis is that prenatal exposure to PFASs might lead to health outcomes in the offspring through epigenetic alterations in utero because epigenetics (i.e., chemical modification of DNA) is an intrinsic biological mechanism that can be affected by extrinsic environmental factors in humans.

DNA methylation is an epigenetic modification that plays a role in embryonic development and cellular differentiation (Breton et al. 2017). It occurs by the addition of a methyl group to a cytosine mostly at cytosine-guanine dinucleotide (CpG) loci and acts like a gene expression switch (Hackett and Surani 2013). Human epidemiological studies, including genome-wide approaches, have indicated that environmental factors such as diet, hormones, stress, drugs, or toxicants (e.g., lead, mercury, or tobacco smoke) during prenatal development influence DNA methylation patterns in children (Breton et al. 2017). Despite a significant impact of PFASs on health outcomes, there were few epidemiological studies of epigenetic effects of PFAS exposure in utero. 
Guerrero-Preston et al. (2010) observed that cord blood PFOA concentrations negatively correlated with cord serum global DNA methylation levels. We also reported that prenatal PFOA exposure was associated with reduced IGF2 methylation in cord blood, which could predict infant ponderal index at birth (Kobayashi et al. 2017).

Genome-wide methylation analyses allow a hypothesis-free assessment of epigenetic alterations in relation to the environmental factors (Christensen and Marsit 2011). To our knowledge, only one study showed an association between maternal PFOA levels and genome-wide DNA methylation using 44 cord blood samples (Kingsley et al. 2017). The objective of the present study was to investigate cord blood DNA methylation changes in association with prenatal exposure to PFASs using the genome-wide approach and to determine CpG loci epigenetically vulnerable to prenatal PFAS exposure.

\section{Materials and Methods}

Study population of the discovery cohort. Participants were enrolled in the Sapporo cohort of the Hokkaido Study on Environment and Children's Health (Kishi et al. 2011, 2013, 2017). Briefly, pregnant women at 23-35 weeks of gestation were recruited at the Toho Hospital (Sapporo, Japan) between 2002 and 2005. Of the 1,796 potentially eligible women, the subjects who decided to participate in the Japanese cord 
blood bank (22\% of those approached) or delivered at another hospital (3\% of those approached) were excluded. Ultimately, 514 pregnant women agreed to participate in this study. At enrollment, a self-administered questionnaire was used to obtain baseline information, including parental demographic characteristics, anthropometric measurements, and lifestyle factors, such as incidence and frequency of maternal smoking and alcohol consumption. Information on pregnancy complications, gestational age, infant gender, and birth size was obtained from medical records. We excluded 17 women from the study due to miscarriage or stillbirth $(n=2)$, relocation $(n=1)$, voluntary withdrawal $(n=7)$, and multiple births $(n=7)$, leaving 497 women who delivered singleton infants.

Exposure assessment in the discovery cohort. Maternal blood samples were collected from the participants between 24 and 41 weeks of gestational age and stored at $-80^{\circ} \mathrm{C}$ prior to analysis. In total, 447 maternal blood samples were available for the exposure assessment. PFOS and PFOA levels were measured in maternal serum by using column-switching liquid chromatography-tandem mass spectrometry (LC-MS/MS) as previously described (Okada et al. 2012; Washino et al. 2009). After excluding of the subjects ( $n=124)$ whose blood samples were obtained after birth due to anemia, data on PFOS and PFOA levels in 323 mother-infant pairs were available. 
450K DNA methylation analysis in the discovery cohort. Umbilical cord blood

samples were taken immediately after birth, and then stored at $-80^{\circ} \mathrm{C}$. In total, 292 cord blood samples were available for DNA methylation analysis. Genomic DNA was extracted from cord blood using a Maxwell ${ }^{\circledR} 16$ DNA Purification Kit (Promega, Madison, WI, USA). DNA methylation at 485,577 CpGs was quantified using the Infinium HumanMethylation 450 BeadChip (Illumina Inc., SanDiego, CA, USA) according to the manufacturer’s protocol (Bibikova et al. 2011; Sandoval et al. 2011). Briefly, DNA (500 ng) was subjected to bisulfite conversion by using a Zymo EZ DNA Methylation Kit (Zymo Research, Irvine, CA, USA) to convert unmethylated cytosine to uracil, leaving methylated cytosine residues unaltered. Bisulfite-treated DNA was then subjected to whole genome amplification, breaking into 300-600-bp nucleotide fragments, and hybridization to the HumanMethylation 450 BeadChip (Illumina Inc.) using a HumanMethylation450 DNA Analysis Kit (Illumina Inc.). After nucleotide extension, iScan (Illumina Inc.) was used to measure signal intensities of each probe on the BeadChip. All analyses were performed by G\&G Science Co., Ltd. (Matsukawa, Fukushima, Japan). Samples were run across five plate batches and were assigned randomized location across plates. To ensure that the experiment was not confounded, we looked at PFAS levels by sample plate and found that there was no significant 
difference of PFAS levels in each of the plates as shown in Supplemental Figure S1.

After quality control (Aryee et al. 2014), signal intensities were normalized using functional normalization (Fortin et al. 2014). Probes with a detection $P$-value $>$ 0.05 in more than $25 \%$ of samples, SNP-affected probes, cross-reactive probes identified by Chen et al. (2013), and probes on sex chromosomes were removed. As a result, 426,413 CpG probes were included in the working set. We applied the ComBat method to adjust methylation data for sample plate to reduce a potential bias due to batch effects (Leek et al. 2012). Beta-values were calculated from signal intensities and used for the subsequent data analyses by using the following equation (Bibikova et al. 2011):

$\beta=$ methylated $/$ (methylated + unmethylated +100 ).

Data analysis. In total, 190 mother-infant pairs, with both exposure and DNA methylation data available, were included in data analysis. PFOS was detected in all samples. For 12 samples with PFOA levels below the detection limit $(0.50 \mathrm{ng} / \mathrm{mL})$, we assigned a value of half the detection limit $(0.25 \mathrm{ng} / \mathrm{mL})$. Because of skewed distributions (Supplemental Figure S2), we transformed PFOS and PFOA concentrations in maternal serum to the $\log _{10}$ scale. Cord blood cell proportion was estimated by the method implemented in the R/Bioconductor package minfi (Bakulski et 
al. 2016). There was no significant correlation between cord blood cell proportions and PFAS levels (Supplemental Table S1). Using limma package in R, robust linear regression analyses (Fox and Weisberg 2011) and empirical Bayesian methods (Smyth 2004) were applied to determine the associations of $\beta$-value at each CpG site with either PFOS or PFOA $\log _{10}$-transformed concentration, adjusted for maternal age, parity, maternal educational levels, maternal blood sampling period, maternal pre-pregnancy BMI, maternal smoking during pregnancy, gestational age, infant sex, and cord blood cell estimates for $\mathrm{CD}^{+}{ }^{+} \mathrm{T}$ cells, $\mathrm{CD} 8^{+} \mathrm{T}$ cells, granulocytes, monocytes, B cell and nucleated red blood cells. Adjustment covariates influencing PFAS levels were selected according to the current results of the present study and from factors previously reported to be associated with cord blood DNA methylation. Statistical analyses were performed using minfi, sva, and limma packages in R ver. 3.3.2 and Bioconductor ver. 3.3.

Genome-wide significance based on a Bonferroni threshold was considered at $P<1.17$ $\times 10^{-7}$. For multiple comparisons, $P$-values were adjusted by the false discovery rate (FDR) to obtain $q$-values. We identified differentially methylated regions (DMRs) associated with PFAS exposures using bumphunter function in R/Bioconductor (Jaffe et al, 2012), a cut-off of 5\% difference in the beta values, and the same models as those in the linear regression analyses. $P$-values were adjusted by the family-wise error rate 
(FWER).

The Spearman’s correlation test, Mann-Whitney U-test, and Kruskal-Wallis test were applied to determine whether maternal and offspring characteristics were associated with PFAS exposure.

Replication study in an independent cohort. Thirty-nine mother-infant pairs that enrolled in the Taiwan Maternal and Infant Cohort Study (TMICS; Huang et al. 2012; Wang et al. 2005) were available for both maternal and cord blood samples and evaluated in the replication study. The concentrations of PFOS and PFOA in blood samples collected from pregnant women during the third trimester at 28-36 weeks were measured using LC-MS/MS as described by Lien et al. (2011). DNA methylation in cord blood samples was assessed using the Illumina Infinium HumanMethylation 450 BeadChip (Kaushal et al. 2017). After the same quality control steps as those used in the discovery cohort, two samples were removed from further analyses. The population characteristics of the replication cohort $(n=37)$ are presented in Supplemental Table S1.

The association between DNA methylation and PFAS concentration was examined using the same robust linear regression models (Fox and Weisberg 2011; Smyth 2004) that were applied in the discovery cohort. There were no smokers during pregnancy in the replication cohort. Due to the small sample size of the replication 
cohort, we used only maternal age, infant sex, and cord blood cell estimates as covariates. Successful replication for differentially methylated positions (DMPs) was defined as having the same direction of effect with those observed in the discovery cohort and $P$-value $<0.05$.

Gene ontology analysis. We identified the enrichment of genes corresponding to the DMPs with $P$-value $<0.001$ in Kyoto Encyclopedia Genes and Genomes (KEGG) pathways (Kanehisa et al. 2002) using missMethyl package in R/Bioconductor (Phipson et al. 2016).

Ethics. The study was conducted with the informed consent of all subjects in the written form. The institutional Ethical Board for human gene and genome studies at the Hokkaido University Graduate School of Medicine and the Hokkaido University Center for Environmental and Health Science approved the study protocol. The Human Ethics Committee of the National Health Research Committee of Taiwan approved the study of the replication cohort.

\section{Results}

\section{Epigenome-wide association study in the discovery cohort}

Maternal and infant characteristics and their relationship to PFOS and PFOA 
concentrations are described in Table 1 . Median ( $25^{\text {th }}$ to $75^{\text {th }}$ percentiles) of PFOS and PFOA concentrations in maternal blood were $5.2 \mathrm{ng} / \mathrm{mL}$ (3.8 to 7.1) and $1.4 \mathrm{ng} / \mathrm{mL}$ (0.9 to 2.1 ), respectively. The average ( \pm SD) age of the mothers was $29.7 \pm 4.8$ years. Of the 190 newborns, 84 (44.2\%) were male. We observed statistically significant differences in both PFOS and PFOA levels by parity, maternal blood sampling periods, and smoking during pregnancy. Additionally, PFOA level was significantly higher among mothers with male infants, and PFOS levels were marginally affected by the educational level.

Figure 1 shows the results of genome-wide analyses of the association between cord blood DNA methylation and prenatal PFOS (Figure 1A) or PFOA (Figure 1B) exposure. The quantile-quantile plot for PFOA exposure did not reveal any significant inflation in the distribution of observed $P$-values (genomic inflation factor: $\lambda=1.02$ ), whereas the distribution of $P$-values for PFOS exposure slightly deviated from the null expected $P$-values ( $\lambda=1.14$ ) as shown in Supplemental Figure S3. The volcano plots (Figure 1) showed imbalance in positive versus negative methylation changes, suggesting global methylation shifts due to PFAS exposure. We examined the location of CpGs associated with PFAS exposure with $P$-value $<0.001$ in gene features and CpG islands. As shown in Supplemental Figure S4, there were statistically 
significant differences associated with PFOS exposure compared with the expected proportions (for gene features, $X^{2} P$-value $=5 \times 10^{-4}$; for CpG islands, $X^{2} P$-value $=$ $4 \times 10^{-4}$ ). Increases in TSS200 and CpG islands were particularly observed.

\section{Differentially methylated positions}

We then focused on the changes at specific regions and found

epigenome-wide significant associations (FDR $q$-value $<0.05$ ) between PFOS exposure and DNA methylation for two CpGs: one located in the intergenic region (IGR) of CXADRP3 (cg02044327), and another mapped to SNAPIN (cg25705526). In addition, significant associations between PFOA exposure and DNA methylation for another two CpGs were found: one located in IGR of AC002480.3 (cg11260715), and another mapped to GPR126 (cg04461802). Top 20 DMPs ranked by the lowest $P$-value for the association with exposure to PFOS and PFOA are shown in Table 2. Among them, four DMPs met the criteria for replication and showed the same direction of effect and $P$-value $<0.05$ in the replication assay: cg16242615 mapped to ZBTB7A for PFOS; cg21876869 located in the IGR of USP2-AS1, cg00173435 mapped to TCP11L2, and cg18901140 located in the IGR of NTN1 for PFOA. The lists of CpGs associated with PFAS exposures with $P$-value $<0.001$ are available in the Supplemental Tables S3 and S4. 


\section{Differentially methylated regions}

Next, we assessed DMRs associated with prenatal PFAS exposures using bumphunter function (Jaffe et al, 2012). We found one region associated with PFOA exposure with FWER $<0.1$ that was located in IGR of ZFP57 and included 21 CpGs. We showed top five regions for PFOS and PFOA exposures ranked by the smallest $P$-value (Table 3). We also compared the direction of methylation changes in the discovery and replication cohorts (Table 4), in which we averaged methylation levels of each site because those were highly correlated (data not shown). A DMR in CYP1E2 was observed for both PFOS and PFOA exposures. Eight of the ten regions showed the same direction of methylation changes in the replication cohort. We also showed the beta coefficients for each CpG site included in the eight DMRs in the discovery and replication cohorts in Supplemental Figure S5.

\section{Gene ontology analysis.}

Lastly, we tested for the enrichment of KEGG pathways (Kanehisa et al. 2002) among the genes with annotated CpGs showing $P$-value $<0.001$. Among the 323 pathways analyzed, 31 and 26 KEGG pathways were significantly enriched after Bonferroni correction among the genes affected by for PFOS and PFOA exposures, respectively. Gene Ontology analyses of the data obtained using 450K chip are known 
to be biased for cancer-related genes (Haper et al. 2013). Human disease pathways, including cancer, were therefore excluded from the list of the pathways affected by PFAS exposures (Supplemental Figure S6). Enrichments in the pathways involved in signal transduction and signal molecules and interactions were observed among the genes affected by both PFOS and PFOA exposures.

\section{Discussion}

Few studies have focused on the epigenetic effects of prenatal exposure to PFASs. In this study, median concentrations of PFOS and PFOA were 5.2 and 1.4 $\mathrm{ng} / \mathrm{mL}$, respectively, which were lower than those reported in the United States (PFOS: 8.2, PFOA: 2.9 ng/mL) (Stein et al. 2012), Canada (PFOS: 16.6, PFOA: $2.1 \mathrm{ng} / \mathrm{mL}$ ) (Monroy et al. 2008), Denmark (PFOS: 21.5, PFOA: 3.7 ng/mL) (Huang et al. 2012), Norway (PFOS: 13, PFOA: 2.2 ng/mL) (Starling et al. 2014), South Korea (PFOS: 9.3, PFOA: $2.6 \mathrm{ng} / \mathrm{mL}$ ) (Lee et al. 2013), and China (PFOS: 6.7, PFOA: 4 ng/mL) (Jiang et al. 2014). Despite the low levels of exposure, we showed suggestive evidence for the presence of CpGs epigenetically vulnerable to PFAS exposure in utero.

We observed potential global methylation shifts resulting from prenatal PFAS exposure (see volcano plots in Figure 1 and Supplemental Figure S3): up-methylation 
for PFOS exposure and down-methylation for PFOA exposure. This was consistent with previous reports for prenatal PFOA exposure (Guerrero-Preston et al. 2010; Kingsley et al. 2017). Two studies in adult populations have suggested a possibility of PFAS exposure effect on global methylation (Leter et al. 2014; Watkins et al. 2014).

We then focused on the changes at specific regions and found four DMPs with FDR < 0.05: cg02044327 (CXADRP3), cg25705526 (SNAPIN), cg11260715 (AC002480.3), and cg04461802 (GPR126) (Figure 1), although these DMPs did not meet the criteria for replication (Table 2). Among 20 DMPs with lowest $P$-values for PFOS and PFOA exposures (Table 2), four DMPs were replicated: cg16242615 (ZBTB7A), cg21876869 (USP2-AS1), cg00173435 (TCP11L2), and cg18901140 (NTN1). ZBTB7A (Zinc finger and BTB domain containing 7A) encodes a proto-oncogenic transcription factor that interacts directly with MBD3 (methyl-CpG-binding domain protein 3) in the nucleus (Choi et al. 2013). TCP11L2 (T-Complex 11 Like 2) codes for the TCP11 like protein. TCP11 plays a role in the process of sperm capacitation and acrosome reactions. USP2-AS1 (USP2 Antisense RNA 1) belongs to the non-coding RNAs. Netrin 1 (NTN1) is a secreted laminin-like protein identified as an axon guidance molecule.

Recently, Breton et al. (2017) have focused on the magnitude of the effect on 
DNA methylation change for health outcome, and similarly, other studies have selected CpGs based on this parameter (Harlid et al. 2017; Kaut et al. 2017). We listed top 20 DMPs ranked by the absolute value of the coefficient with $P<0.001$ in Supplemental Table S5. Among them, three DMPs met the criteria for replication: cg01788773 located in the IGR of LOC286083, cg23625390 mapped to SCAPER, and cg02546416 located in the IGR of RP5-1086L22.1. Although the associations were not statistically significant in the replication cohort, 16 out of 20 DMPs for PFOS exposure notably showed increased methylation changes in both discovery and replication cohorts (Supplemental Table S5). In particular, PKP3 (plakophilin 3) harbored four sites with methylation changes in a coherent direction.

Next, we explored DMRs that are potentially more informative than individual CpG sites (Solomon et al. 2017). We found one down-methylated region with FWER $<0.1$, which was located in the IGR of ZFP57 (ZFP57 Zinc Finger Protein) (Table 4). ZFP57 is necessary for maintaining repressive epigenetic modifications at imprinting control regions (Riso et al. 2016). We observed down-methylation of this region in the replication cohort (Table 4 and Supplemental Figure S6). In addition to a DMR in ZFP57, we reported six DMRs in CYP2E1, SMAD3, SLC17A9, GFPT2, DUSP22, and TCERG1L that showed the same direction of methylation change in the 
replication cohort (Table 4 and Supplemental Figure S6). Among them, methylation of SMAD3 (SMAD Family Member 3) at birth has been previously linked to asthma in children of asthmatic mothers (DeVries et al. 2016). Cord blood DNA methylation of GFPT2 (Glutamine-Fructose-6-Phosphate Transaminase 2) was associated with adiposity in childhood (Kresovich et al. 2017). Furthermore, methylations of CYP2E1 (Cytochrome P450 Family 2 Subfamily E Member 1), DUSP2 (Dual Specificity Phosphatase 2), and TCERG1L (transcription elongation regulator 1-like) were associated with rheumatoid arthritis (Mok et al. 2017) and colon tumors (Bae et al. 2014). Additionally, PFOS inhibited the oxidation reaction of CYP2E1 in vitro (Narimatu et al. 2011).

Previously, our studies from the same cohort have shown that prenatal exposure to PFASs affected offspring development, such as birth size (Washino et al. 2009; Kobayashi et al. 2017), cord adipokine levels; biomarkers of metabolic function (Minatoya et al. 2017), and neurodevelopment (Goudarzi et al. 2016). In addition, prenatal PFAS exposure was associated with hormone levels at birth (Kato et al. 2016; Itoh et al. 2016; Goudarzi et al. 2017) and immune function (Okada et al. 2012, 2014). Methylation changes might link health outcomes to PFAS exposure. Given the various effects of prenatal PFAS exposure on health outcomes, it is plausible that methylation 
changes occur in various signaling pathways. Gene ontology analysis showed that differentially methylated genes were enriched in multiple KEGG pathways (Supplemental Figure 6), including signal transduction, signaling molecules and interaction, endocrine system, and immune system. However, in this assay we used genes with annotated CpGs with $P$-value $<0.001$, i.e., not achieving epigenome-wide significance. It remains to be seen whether identified DNA methylation changes are functionally relevant.

The only previous study of epigenetic effects of PFOA exposure using the genome-wide analysis reported cord blood DNA methylation differences at seven CpGs in three genes, RASA3, UCK1, and OPRD1, between subjects with high ( $n=22$, median: $15 \mathrm{ng} / \mathrm{mL}$, range: $12-26 \mathrm{ng} / \mathrm{mL}$ ) and low (n=22, median: $2.5 \mathrm{ng} / \mathrm{mL}$, range: 1.1-3.1 ng $/ \mathrm{mL}$ ) maternal PFOA levels at up to16 weeks of pregnancy in the United States (Kingsley et al. 2017). These changes were not replicated in our study. As described above, the median ( $25^{\text {th }}$ to $75^{\text {th }}$ percentiles) of PFOA concentration in our discovery cohort was $1.4 \mathrm{ng} / \mathrm{mL}$ (0.9 to 1.8 ), which was still lower than the level in low PFOA exposure group in the study of Kingsley et al. (2017). The differences in PFOA levels, study design, and blood sampling period may explain these discrepancies. It is possible that future studies with similar exposure levels or larger sample sizes will find a more 
significant overlap between our results and findings of others.

Methylation changes derived from the exposures to PFOS and PFOA were different. As described above, prenatal PFAS exposures were associated with various health outcomes. Although the mechanism of this association has yet to be fully elucidated, Gyllenhammar et al. (2017) and Halldorosson et al. (2012) have suggested at least three potential pathways: firstly, PFASs may impair estrogen system; secondly, PFASs can activate the peroxisome proliferator-activated receptors (PPARs) involved in lipid metabolism; thirdly, PFASs may interact with thyroid hormones. We have reported that PFOS and PFOA differentially affected health outcomes related to these pathways. Prenatal exposure to PFOS, but not PFOA, was negatively associated with the levels of maternal fatty acids (Kishi et al. 2015), possibly disrupted both maternal and infant thyroid hormone levels (Kato et al. 2016), and showed an inverse relationship with cord blood levels of glucocorticoids (Goudarzi et al. 2017). Dehydroepiandrosterone level was positively associated with the exposure to PFOS and negatively associated with the exposure to PFOA (Goudarzi et al. 2017). PFOS and PFOA showed both positive and inverse associations with the levels of several reproductive and steroid hormones (Ito et al. 2016). The observed different potencies and modes of action may partly account for the distinct patterns of methylation changes. The differences in PFOS and PFOA 
concentrations and/or placental permeability could be an alternative explanation.

The limitations of this study should be considered. We measured prenatal PFAS exposure levels and cord blood genome-wide DNA methylation in a prospective birth cohort and identified DNA methylation changes significantly associated with the exposure to relatively low levels of PFASs in utero. However, there remains a possibility that residual confounding factors may bias or influence the results. We analyzed DNA methylation in cord blood, which may not be representative of the methylation levels in target tissues of interest. In this study, we included participants for whom both maternal and cord blood samples were available, thus limiting the scope only to mothers who delivered vaginally. It is thus possible that relatively healthier children were included in our analysis, and we may have underestimated the effects of PFAS exposure. Additionally, we excluded subjects whose blood samples were obtained after delivery ( $n=124)$. The excluded subjects had educational levels, annual household income levels, and alcohol consumption status similar to those of participants in this study, but higher percentage of multiparous and smoking during pregnancy, which may suggest a possibility of selection bias. Lastly, because of the limited sample size, the replication assay was likely underpowered. As a result, DMPs replicated based on $P$-values were very limited, whereas the directions of effect were replicated in a number 
of CpG sites, including DMRs.

\section{Conclusion}

In this epigenome-wide study, we suggested that even relatively low levels of prenatal exposure to PFASs impacted DNA methylation status at birth. Further study is needed to examine the persistence of DNA methylation changes due to prenatal exposure throughout life, and the associations of these changes with health outcomes causally linked to PFAS exposure in longitudinal studies.

\section{Acknowledgments}

The authors acknowledge all the participants and staff in this study. This study was funded by the Environment Research and Technology Development Fund from the Japanese Ministry of the Environment (No. 5-1454), a Grant-in-Aid for Health Science Research from the Japanese Ministry of Health, Labor and Welfare (No. 201624002B; 17932352); and a Grant-in-Aid for Scientific Research from the Japanese Ministry of

Education, Culture, Sports, Science and Technology (No. 25253050; 16K15352;

16H02645). Funding for the Maternal and Infant Cohort Study in Taiwan was provided by the National Health Research Institute, Miaoli, Taiwan (Grant No. EO-091-PP-01; 
Miura et al.

EM-106-PP-05), and the Ministry of Science and Technology, Taiwan (MOST

105-2314-B-400-001). The authors declare they have no actual or potential competing

financial interests. 


\section{References}

Abbott BD, Wolf CJ, Schmid JE, Das KP, Zehr RD, Helfant L, et al. 2007. Perfluorooctanoic acid induced developmental toxicity in the mouse is dependent on expression of peroxisome proliferator activated receptor-alpha. Toxicological Sciences 98:571-581.

Apelberg BJ, Witter FR, Herbstman JB, Calafat AM, Halden RU, Needham LL, et al. 2007. Cord serum concentrations of perfluorooctane sulfonate (pfos) and perfluorooctanoate (pfoa) in relation to weight and size at birth. Environmental Health Perspectives 115:1670-1676.

Aryee MJ, Jaffe AE, Corrada-Bravo H, Ladd-Acosta C, Feinberg AP, Hansen KD, et al. 2014. Minfi: A flexible and comprehensive bioconductor package for the analysis of infinium DNA methylation microarrays. Bioinformatics (Oxford, England) 30:1363-1369.

Bae JH, Park J, Yang KM, Kim TO, Yi JM; IBD study group of Korean Association for Study of Intestinal Diseases (KASID). Molecular Medicine Reports 9:725-9.

Bakulski KM, Feinberg JI, Andrews SV, Yang J, Brown S, S LM, et al. 2016. DNA methylation of cord blood cell types: Applications for mixed cell birth studies. Epigenetics 11:354-362.

Bibikova M, Barnes B, Tsan C, Ho V, Klotzle B, Le JM, et al. 2011. High density DNA methylation array with single cpg site resolution. Genomics 98:288-295.

Breton CV, Marsit CJ, Faustman E, Nadeau K, Goodrich JM, Dolinoy DC, et al. 2017. Small-magnitude effect sizes in epigenetic end points are important in children's environmental health studies: The children's environmental health and disease prevention research center's epigenetics working group. Environmental Health Perspectives 125:511-526.

Chen YA, Lemire M, Choufani S, Butcher DT, Grafodatskaya D, Zanke BW, et al. 2013. Discovery of cross-reactive probes and polymorphic cpgs in the illumina infinium humanmethylation450 microarray. Epigenetics 8:203-209.

Christensen BC, Marsit CJ. 2011. Epigenomics in environmental health. Frontiers in Genetics 2:84.

Choi WI, Jeon BN, Yoon JH, Koh DI, Kim MH, Yu MY, et al. 2013. The proto-oncoprotein FBI-1 interacts with MBD3 to recruit the Mi-2/NuRD-HDAC complex and BCoR and to silence p21WAF/CDKN1A by DNA methylation. Nucleic Acids Research 41:6403-6420.

DeVries A, Wlasiuk G, Miller SJ, Bosco A, Stern DA, Lohman IC, et al. 2016.

Epigenome-wide analysis links SMAD3 methylation at birth to asthma in children of asthmatic mothers. Journal of Allergy Clinical Immunology 140:534-542.

DeWitt JC, Peden-Adams MM, Keller JM, Germolec DR. 2012. Immunotoxicity of 
perfluorinated compounds: Recent developments. Toxicologic Pathology 40:300-311.

Fortin JP, Labbe A, Lemire M, Zanke BW, Hudson TJ, Fertig EJ, et al. 2014. Functional normalization of $450 \mathrm{k}$ methylation array data improves replication in large cancer studies. Genome Biology 15:503.

Fox J, Weisberg S. 2011. Robust regression in r. Thousand Oaks, CA.:Sage.

Goudarzi H, Nakajima S, Ikeno T, Sasaki S, Kobayashi S, Miyashita C, et al. 2016. Prenatal exposure to perfluorinated chemicals and neurodevelopment in early infancy: The Hokkaido study. The Science of the Total Environment 541:1002-1010.

Goudarzi H, Araki A, Itoh S, Sasaki S, Miyashita C, Mitsui T, et al. 2017. The association of prenatal exposure to perfluorinated chemicals with glucocorticoid and androgenic hormones in cord blood samples: The Hokkaido study. Environmental Health Perspectives 125:111-118.

Grandjean P, Andersen EW, Budtz-Jorgensen E, Nielsen F, Molbak K, Weihe P, et al. 2012. Serum vaccine antibody concentrations in children exposed to perfluorinated compounds. Jama 307:391-397.

Gyllenhammar I, Diderholm B, Gustafsson J, Berger U, Ridefelt P, Benskin JP, et al. 2017. Perfluoroalkyl acid levels in first-time mothers in relation to offspring weight gain and growth. Environment International 111:191-199.

Hackett JA, Surani MA. 2013. DNA methylation dynamics during the mammalian life cycle. Philosophical Transactions of the Royal Society of London Series B, Biological Sciences 368:20110328.

Halldorsson TI, Rytter D, Haug LS, Bech BH, Danielsen I, Becher G, et al. 2012. Prenatal exposure to perfluorooctanoate and risk of overweight at 20 years of age: A prospective cohort study. Environmental Health Perspectives 120:668-673.

Harlid S, Adgent M, Jefferson WN, Panduri V, Umbach DM, Xu Z, et al. 2017. Soy formula and epigenetic modifications: Analysis of vaginal epithelial cells from infant girls in the ifed study. Environmental Health Perspectives 125:447-452.

Harper KN, Peters BA, Gamble MV. 2013. Batch effects and pathway analysis: two potential perils in cancer studies involving DNA methylation array analysis. Cancer Epidemiology Biomarkers and Prevention 22:1052-60.

Huang PC, Su PH, Chen HY, Huang HB, Tsai JL, Huang HI, et al. 2012. Childhood blood lead levels and intellectual development after ban of leaded gasoline in taiwan: A 9-year prospective study. Environment International 40:88-96.

Hayes AF. 2013. Introduction to Mediation, Moderation, and Conditional Process Analysis: A Regression-Based Approach. The Guilford Press (New York, NY)

Inoue K, Okada F, Ito R, Kato S, Sasaki S, Nakajima S, et al. 2004. Perfluorooctane 
sulfonate (pfos) and related perfluorinated compounds in human maternal and cord blood samples: Assessment of pfos exposure in a susceptible population during pregnancy. Environmental Health Perspectives 112:1204-1207.

Itoh S, Araki A, Mitsui T, Miyashita C, Goudarzi H, Sasaki S, et al. 2016. Association of perfluoroalkyl substances exposure in utero with reproductive hormone levels in cord blood in the Hokkaido study on environment and children's health. Environment international 94:51-59.

Jaffe AE, Murakami P, Lee H, Leek JT, Fallin MD, Feinberg AP, Irizarry RA, 2012. Bump hunting to identify differentially methylated regions in epigenetic epidemiology studies. International journal of epidemiology 41:200-209

Jiang W, Zhang Y, Zhu L, Deng J. 2014. Serum levels of perfluoroalkyl acids (pfaas) with isomer analysis and their associations with medical parameters in chinese pregnant women. Environment International 64:40-47.

Johansson N, Fredriksson A, Eriksson P. 2008. Neonatal exposure to perfluorooctane sulfonate (pfos) and perfluorooctanoic acid (pfoa) causes neurobehavioural defects in adult mice. Neurotoxicology 29:160-169.

Johansson N, Eriksson P, Viberg H. 2009. Neonatal exposure to pfos and pfoa in mice results in changes in proteins which are important for neuronal growth and synaptogenesis in the developing brain. Toxicological Sciences 108:412-418.

Kanehisa M, Goto S, Kawashima S, Nakaya A. 2002. The kegg databases at genomenet. Nucleic Acids Research 30:42-46.

Kato S, Itoh S, Yuasa M, Baba T, Miyashita C, Sasaki S, et al. 2016. Association of perfluorinated chemical exposure in utero with maternal and infant thyroid hormone levels in the sapporo cohort of Hokkaido study on the environment and children's health. Environmental Health and Perspective Medicine 21:334-344.

Kaushal A, Zhang H, Karmaus WJJ, Everson TM, Marsit CJ, Karagas MR, et al. 2017. Genome-wide DNA methylation at birth in relation to in utero arsenic exposure and the associated health in later life. Environmental Health : a global access science source $16: 50$.

Kaut O, Schmitt I, Tost J, Busato F, Liu Y, Hofmann P, et al. 2017. Epigenome-wide DNA methylation analysis in siblings and monozygotic twins discordant for sporadic parkinson's disease revealed different epigenetic patterns in peripheral blood mononuclear cells. Neurogenetics 18:7-22.

Kingsley SL, Kelsey KT, Butler R, Chen A, Eliot MN, Romano ME, et al. 2017. Maternal serum pfoa concentration and DNA methylation in cord blood: A pilot study. Environmental Research 158:174-178. 
Kishi R, Sasaki S, Yoshioka E, Yuasa M, Sata F, Saijo Y, et al. 2011. Cohort profile: The Hokkaido study on environment and children's health in japan. International Journal of Epidemiology 40:611-618.

Kishi R, Kobayashi S, Ikeno T, Araki A, Miyashita C, Itoh S, et al. 2013. Ten years of progress in the Hokkaido birth cohort study on environment and children's health: Cohort profile--updated 2013. Environmental Health and Perspective Medicine 18:429-450.

Kishi R, Nakajima T, Goudarzi H, Kobayashi S, Sasaki S, Okada E, et al, 2015. The Association of Prenatal Exposure to Perfluorinated Chemicals with Maternal Essential and Long-Chain Polyunsaturated Fatty Acids during Pregnancy and the Birth Weight of Their Offspring: The Hokkaido Study. Environmental Health Perspectives 123:1038-45.

Kishi R, Araki A, Minatoya M, Hanaoka T, Miyashita C, Itoh S, et al. 2017. The Hokkaido birth cohort study on environment and children's health: Cohort profile-updated 2017. Environmental Health and Preventive Medicine 22.

Kobayashi S, Azumi K, Goudarzi H, Araki A, Miyashita C, Kobayashi S, et al. 2017. Effects of prenatal perfluoroalkyl acid exposure on cord blood igf2/h19 methylation and ponderal index: The Hokkaido study. Journal of Exposure Science \& Environmental epidemiology 27:251-259.

Kresovich JK, Zheng Y, Cardenas A, Joyce BT, Rifas-Shiman SL, Oken E, et al. 2017. Cord blood DNA methylation and adiposity measures in early and mid-childhood. Clinical Epigenetics 9:86.

Lau C, Anitole K, Hodes C, Lai D, Pfahles-Hutchens A, Seed J. 2007. Perfluoroalkyl acids: A review of monitoring and toxicological findings. Toxicological Sciences 99:366-394.

Lee YJ, Kim MK, Bae J, Yang JH. 2013. Concentrations of perfluoroalkyl compounds in maternal and umbilical cord sera and birth outcomes in korea. Chemosphere 90:1603-1609.

Leter G, Consales C, Eleuteri P, Uccelli R, Specht IO, Toft G, et al. 2014. Exposure to perfluoroalkyl substances and sperm DNA global methylation in Arctic and European populations. Environmental and Molecular Mutagenesis 55:591-600.

Leek JT, Johnson WE, Parker HS, Jaffe AE, Storey JD. 2012. The sva package for removing batch effects and other unwanted variation in high-throughput experiments. Bioinformatics 28:882-883.

Liao CY, Li XY, Wu B, Duan S, Jiang GB. 2008. Acute enhancement of synaptic transmission and chronic inhibition of synaptogenesis induced by perfluorooctane sulfonate through mediation of voltage-dependent calcium channel. Environmental Science \& Technology 
42:5335-5341.

Liao CY, Cui L, Zhou QF, Duan SM, Jiang GB. 2009. Effects of perfluorooctane sulfonate on ion channels and glutamate-activated current in cultured rat hippocampal neurons. Environmental Toxicology and Pharmacology 27:338-344.

Lien GW, Wen TW, Hsieh WS, Wu KY, Chen CY, Chen PC. 2011. Analysis of perfluorinated chemicals in umbilical cord blood by ultra-high performance liquid chromatography/tandem mass spectrometry. Journal of Chromatography B 879:641-646.

Luebker DJ, York RG, Hansen KJ, Moore JA, Butenhoff JL. 2005. Neonatal mortality from in utero exposure to perfluorooctanesulfonate (pfos) in sprague-dawley rats: Dose-response, and biochemical and pharamacokinetic parameters. Toxicology 215:149-169.

Minatoya M, Itoh S, Miyashita C, Araki A, Sasaki S, Miura R, et al. 2017. Association of prenatal exposure to perfluoroalkyl substances with cord blood adipokines and birth size: The Hokkaido study on environment and children's health. Environmental Research 156:175-182.

Mok A, Rhead B, Holingue C, Shao X, Quach HL, Quach D et al. 2017. Hypomethylation of CYP2E1 and DUSP22 promoters associated with disease activity and erosive disease among rheumatoid arthritis patients. Arthritis Rheumatolgy (in press) doi:10.1002/art.40408

Monroy R, Morrison K, Teo K, Atkinson S, Kubwabo C, Stewart B, et al. 2008. Serum levels of perfluoroalkyl compounds in human maternal and umbilical cord blood samples. Environmental Research 108:56-62.

Narimatsu S, Nakanishi R, Hanioka N, Saito K, Kataoka H. 2011 Characterization of inhibitory effects of perfluorooctane sulfonate on human hepatic cytochrome P450 isoenzymes: focusing on CYP2A6. Chemico-Biological Interactions 194:120-126.

Okada E, Sasaki S, Saijo Y, Washino N, Miyashita C, Kobayashi S, et al. 2012. Prenatal exposure to perfluorinated chemicals and relationship with allergies and infectious diseases in infants. Environmental Research 112:118-125.

Okada E, Sasaki S, Kashino I, Matsuura H, Miyashita C, Kobayashi S, et al. 2014. Prenatal exposure to perfluoroalkyl acids and allergic diseases in early childhood. Environment International 65:127-134.

Olsen GW, Burris JM, Ehresman DJ, Froehlich JW, Seacat AM, Butenhoff JL, et al. 2007. Half-life of serum elimination of perfluorooctanesulfonate,perfluorohexanesulfonate, and perfluorooctanoate in retired fluorochemical production workers. Environmental Health Perspectives 115:1298-1305. 
Olsen GW, Butenhoff JL, Zobel LR. 2009. Perfluoroalkyl chemicals and human fetal development: An epidemiologic review with clinical and toxicological perspectives. Reproductive toxicology 27:212-230.

Phipson B, Maksimovic J, Oshlack A. 2016. Missmethyl: An r package for analyzing data from illumina's humanmethylation450 platform. Bioinformatics 32:286-288.

Riso V, Cammisa M, Kukreja H, Anvar Z, Verde G, Sparago A, et al. 2016. ZFP57 maintains the parent-of-origin-specific expression of the imprinted genes and differentially affects non-imprinted targets in mouse embryonic stem cells. Nucleic Acids Research. 44:8165-78.

Sandoval J, Heyn H, Moran S, Serra-Musach J, Pujana MA, Bibikova M, et al. 2011. Validation of a DNA methylation microarray for 450,000 cpg sites in the human genome. Epigenetics 6:692-702.

Seacat AM, Thomford PJ, Hansen KJ, Clemen LA, Eldridge SR, Elcombe CR, et al. 2003. Sub-chronic dietary toxicity of potassium perfluorooctanesulfonate in rats. Toxicology 183:117-131.

Smyth GK. 2004. Linear models and empirical bayes methods for assessing differential expression in microarray experiments. Statistical Applications in Genetics and Molecular Biology 3:Article3.

Solomon O, Yousefi P, Huen K, Gunier RB, Escudero-Fung M, et al 2017.

Prenatal phthalate exposure and altered patterns of DNA methylation in cord blood. Environmental Molecular Mutagenesis 58:398-410.

Starling AP, Engel SM, Whitworth KW, Richardson DB, Stuebe AM, Daniels JL, et al. 2014. Perfluoroalkyl substances and lipid concentrations in plasma during pregnancy among women in the norwegian mother and child cohort study. Environment International 62:104-112.

Stein CR, Wolff MS, Calafat AM, Kato K, Engel SM. 2012. Comparison of polyfluoroalkyl compound concentrations in maternal serum and amniotic fluid: A pilot study. Reproductive Toxicology 34:312-316.

Thibodeaux JR, Hanson RG, Rogers JM, Grey BE, Barbee BD, Richards JH, et al. 2003. Exposure to perfluorooctane sulfonate during pregnancy in rat and mouse. I: Maternal and prenatal evaluations. Toxicological Sciences 74:369-381.

Vanden Heuvel JP, Thompson JT, Frame SR, Gillies PJ. 2006. Differential activation of nuclear receptors by perfluorinated fatty acid analogs and natural fatty acids: a comparison of human, mouse, and rat peroxisome proliferator-activated receptor-alpha, -beta, and -gamma, liver X receptor-beta, and retinoid X receptor-alpha. Toxicology Sciences 92:476-89. 
Wang SL, Su PH, Jong SB, Guo YL, Chou WL, Papke O. 2005. In utero exposure to dioxins and polychlorinated biphenyls and its relations to thyroid function and growth hormone in newborns. Environmental Health Perspectives 113:1645-1650.

Washino N, Saijo Y, Sasaki S, Kato S, Ban S, Konishi K, et al. 2009. Correlations between prenatal exposure to perfluorinated chemicals and reduced fetal growth. Environmental Health Perspectives 117:660-667.

Watkins DJ, Wellenius GA, Butler RA, Bartell SM, Fletcher T, Kelsey KT. 2014. Associations between serum perfluoroalkyl acids and LINE-1 DNA methylation. Environment International 63:71-76 
Table 1 Characteristics of study population of the discovery cohort $(n=190)$.

\begin{tabular}{ccccc}
\hline & & & PFOS $(\mathbf{n g} / \mathbf{m L})$ & PFOA (ng/mL) \\
\cline { 3 - 5 } & & Mean \pm SD & Median & Median \\
\hline
\end{tabular}

\section{Concentration in maternal blood}

190

\section{Maternal characteristics}

Maternal age (year) ${ }^{\mathrm{a}}$ 190

$29.7 \pm 4.8$

$5.2(3.8,7.1)$

$1.4(0.9,2.1)$

Pre-pregnancy BMI $\left(\mathrm{kg} / \mathrm{m}^{2}\right)^{\mathrm{a}}$

$19021.2 \pm 3.1 \quad \rho=-0.018 \quad 0.803 \quad \rho=-0.056 \quad 0.444$

Parity (times) ${ }^{\mathrm{b}}$

$\begin{array}{lcc}0 & 104 & 54.7 \\ \geq 1 & 86 & 45.2\end{array}$

54.7

$5.7(4.2,8.0)$

0.002

$1.6(1.2,2.4) \quad<0.001$

$\geq 1$

45.2

$4.7(3.1,6.2)$

$1.0(0.7,1.5)$

Blood sampling period ${ }^{\mathrm{c}}$

$\begin{array}{lll}<28 \text { weeks } & 74 & 38.9 \\ \text { 28-36 weeks } & 47 & 24.7 \\ \geqq 36 \text { weeks } & 69 & 36.3\end{array}$

$$
5.8(4.6,7.5)
$$

$<0.001$

$1.7(1.2,2.3)$

0.004

$\geqq 36$ weeks

36.3

$5.6(4.0,8.5)$

$4.6(2.8,5.7)$

$1.3(0.8,1.8)$

$1.2(0.8,1.8)$

Educational level (year) ${ }^{\mathrm{b}}$

$\begin{array}{rrr}\leq 12 & 89 & 46.8 \\ >12 & 101 & 53.2\end{array}$

46.8

$5.2(4.1,7.0)$

0.966

$1.3(0.8,1.8)$

0.072

Annual household income (million yen) ${ }^{c}$

$<3$
$3-5$

missing

38

20.0

50.0

21.1

7.9

1.1

$5.3(3.6,7.4)$

$1.5(1.0,2.3)$

Smoking during pregnancy ${ }^{\mathrm{b}}$

$\begin{array}{lrr}\text { No } & 157 & 82.6 \\ \text { Yes } & 33 & 17.4\end{array}$

82.6

17.4

$5.4(3.9,8.0)$

0.878

$5.1(3.5,7.0)$

$5.4(4.2,6.9)$

$5.1(3.0,8.8)$

$7.8(4.5,11.1)$

$\begin{array}{lcc}\text { No } & 130 & 68.4 \\ \text { Yes } & 60 & 31.6\end{array}$

$5.2(3.9,7.2)$

0.954

$5.3(3.7,7.1)$

$1.4(0.8,2.2)$

0.541

$1.4(0.9,1.8)$

$1.4(0.9,2.1)$

$2.3(0.8,2.4)$

Infant characteristics

$\begin{array}{llcr}\text { Sex }^{\mathrm{b}} & \text { Male } & 84 & 44.2 \\ & \text { Female } & 106 & 55.8\end{array}$

Gestational age (week) ${ }^{\mathrm{a}}$

190

$39.9 \pm 1.0$

$\begin{array}{cc}5.1(3.3,7.0) & 0.117 \\ 5.4(4.1,7.5) & \\ \rho=0.031 & 0.675\end{array}$

$1.6(1.0,2.4)$

0.025

$1.3(0.8,1.9)$

$\rho=0.093$

0.203 
Miura et al.

Birth weight (g) ${ }^{\mathrm{a}}$

190

$3131 \pm 330$

$\rho=-0.122$

0.095

$\rho=-0.118$

0.104

${ }^{\mathrm{a}}$ Spearman's correlation test ( $\left.\rho\right)$, ${ }^{\mathrm{b}}$ Mann-Whitney U-test, ${ }^{\mathrm{c}}$ Kruskal-Wallis test 
Table 2. Top $20 \mathrm{CpGs}$ ranked by the smallest $P$-value from the epigenome-wide analysis of the association between prenatal PFAS exposure and cord blood DNA methylation.

\begin{tabular}{|c|c|c|c|c|c|c|c|c|}
\hline \multirow{2}{*}{ Probe ID } & \multirow{2}{*}{ Gene } & \multirow{2}{*}{ Chr } & \multirow{2}{*}{ Feature $^{a}$} & \multicolumn{2}{|c|}{ Discovery cohort } & \multicolumn{2}{|c|}{ Replication cohort } & \multirow{2}{*}{ Replicated $^{\mathrm{d}}$} \\
\hline & & & & $\operatorname{Coef}^{b}$ & P-Value & Coef $^{\mathrm{b}}$ & P-Value & \\
\hline \multicolumn{9}{|l|}{$\log _{10}(\mathrm{PFOS})$} \\
\hline cg25705526 & SNAPIN & 1 & TSS200 & 0.020 & $1.48 \mathrm{E}-07^{F D R, d}$ & -0.006 & 0.425 & \\
\hline cg04928693 & MTX1 & 1 & TSS1500 & 0.024 & 7.19E-07 & -0.003 & 0.737 & \\
\hline $\operatorname{cg} 18155888^{c}$ & MORN1 & 1 & Body & 0.017 & 4.32E-06 & 0.023 & 0.277 & \\
\hline cg17086204 & PLA2G5 & 1 & 3'UTR & 0.025 & 5.56E-06 & 0.000 & 0.955 & \\
\hline cg10504365 & TRIM67 & 1 & TSS1500 & 0.032 & 5.84E-06 & -0.022 & 0.003 & \\
\hline $\operatorname{cg} 01889773^{c}$ & GPC1 & 2 & Body & 0.030 & 6.92E-07 & 0.008 & 0.538 & \\
\hline cg16845265 & KLHL29 & 2 & IGR & 0.065 & 8.73E-07 & -0.019 & 0.528 & \\
\hline cg25808157 & GREB1 & 2 & Body & 0.034 & 3.13E-06 & -0.002 & 0.926 & \\
\hline cg21969395 & $L R A T$ & 4 & TSS200 & 0.016 & 8.23E-06 & -0.001 & 0.870 & \\
\hline cg22953687 & ZFYVE28 & 4 & Body & 0.025 & 8.25E-06 & -0.018 & 0.117 & \\
\hline cg12215478 & SRPK1 & 6 & Body & 0.008 & 6.93E-06 & -0.002 & 0.421 & \\
\hline $\operatorname{cg} 14369981^{c}$ & $L M X 1 B$ & 9 & IGR & 0.037 & 2.81E-06 & 0.007 & 0.705 & \\
\hline $\operatorname{cg} 17918227^{c}$ & CADM1 & 11 & Body & 0.012 & 7.56E-06 & 0.005 & 0.336 & \\
\hline $\operatorname{cg} 14120075^{c}$ & TFDP1 & 13 & Body & 0.031 & 3.94E-06 & 0.008 & 0.626 & \\
\hline $\operatorname{cg} 03097541^{c}$ & ZNF213 & 16 & 5'UTR & 0.006 & 3.38E-06 & 0.002 & 0.131 & \\
\hline cg01718742 & CDH8 & 16 & TSS200 & 0.003 & 4.06E-06 & -0.001 & 0.651 & \\
\hline $\operatorname{cg} 02044327^{c}$ & CXADRP3 & 18 & IGR & 0.078 & $4.06 \mathrm{E}-08^{F D R, d}$ & 0.036 & 0.142 & \\
\hline $\operatorname{cg} 16242615^{c}$ & ZBTB7A & 19 & 5'UTR & 0.037 & $4.54 \mathrm{E}-06$ & 0.035 & 0.028 & $\checkmark$ \\
\hline cg15815607 & HM13 & 20 & Body & 0.053 & 4.57E-06 & -0.008 & 0.645 & \\
\hline cg12700033 & YWHAH & 22 & TSS200 & 0.006 & 7.41E-06 & 0.000 & 0.958 & \\
\hline \multicolumn{9}{|l|}{$\log _{10}(\mathrm{PFOA})$} \\
\hline cg23049737 & $R E R E$ & 1 & 3'UTR & 0.011 & $1.43 \mathrm{E}-05$ & -0.009 & 0.056 & \\
\hline $\operatorname{cg} 00567854^{c}$ & BTG2 & 1 & TSS1500 & -0.025 & $1.48 \mathrm{E}-05$ & -0.009 & 0.214 & \\
\hline cg10403518 & SLC9A4 & 2 & Body & 0.038 & 1.75E-06 & -0.014 & 0.083 & \\
\hline cg22325921 & $D U X 2$ & 4 & IGR & -0.020 & $9.90 \mathrm{E}-06$ & 0.002 & 0.719 & \\
\hline $\operatorname{cg} 05158146^{c}$ & MIR4460 & 5 & IGR & -0.010 & 6.75E-06 & 0.000 & 0.931 & \\
\hline $\operatorname{cg} 23917868^{c}$ & MIR145 & 5 & TSS200 & -0.020 & $1.76 \mathrm{E}-05$ & -0.006 & 0.289 & \\
\hline cg04461802 & GPR126 & 6 & 5'UTR & -0.046 & $1.65 \mathrm{E}-07^{F D R, d}$ & 0.008 & 0.456 & \\
\hline $\operatorname{cg} 11260715^{c}$ & AC002480.3 & 7 & IGR & -0.008 & $2.32 \mathrm{E}-08^{F D R, d}$ & -0.002 & 0.247 & \\
\hline $\operatorname{cg} 12105980^{c}$ & EN2 & 7 & IGR & -0.023 & $1.24 \mathrm{E}-05$ & -0.008 & 0.220 & \\
\hline cg13951074 & DMRT2 & 9 & IGR & 0.010 & 5.09E-07 & 0.000 & 0.888 & \\
\hline $\operatorname{cg} 07661167^{\mathrm{C}}$ & ZNF33BP1 & 10 & IGR & 0.032 & 1.62E-06 & 0.015 & 0.065 & \\
\hline
\end{tabular}




\begin{tabular}{|c|c|c|c|c|c|c|c|}
\hline cg01486146 & $P A X 2$ & 10 & IGR & -0.014 & $1.50 \mathrm{E}-05$ & 0.005 & 0.088 \\
\hline cg21876869 & USP2-AS1 & 11 & IGR & -0.027 & $1.64 \mathrm{E}-06$ & -0.024 & 0.000 \\
\hline cg17114584 & IRF7 & 11 & Body & -0.047 & 9.01E-06 & 0.001 & 0.928 \\
\hline $\operatorname{cg} 00173435^{c}$ & TCP11L2 & 12 & TSS200 & 0.006 & 8.52E-07 & 0.003 & 0.001 \\
\hline $\operatorname{cg} 16475925^{c}$ & SPG7 & 16 & TSS200 & 0.003 & $1.82 \mathrm{E}-05$ & 0.002 & 0.095 \\
\hline cg00897875 & МАРЗК14 & 17 & 5'UTR & -0.006 & $9.16 \mathrm{E}-07$ & 0.004 & 0.077 \\
\hline $\operatorname{cg} 18901140^{c}$ & NTN1 & 17 & IGR & -0.036 & 2.66E-06 & -0.029 & 0.000 \\
\hline $\operatorname{cg} 01426818^{c}$ & $C B X 4$ & 17 & IGR & -0.012 & $5.20 \mathrm{E}-06$ & -0.002 & 0.720 \\
\hline $\operatorname{cg} 18002862^{c}$ & $R A P 1 G A P 2$ & 17 & Body & -0.008 & $1.22 \mathrm{E}-05$ & -0.002 & 0.146 \\
\hline
\end{tabular}

Abbreviations: Chr, chromosome; IGR, intergenic region; TSS, transcription start site; TSS200, 200 bases from TSS; TSS1500, 1500 bases from TSS; body, gene body; UTR, untranslated region.

${ }^{\mathrm{a}}$ Gene feature category of the methylation locus.

${ }^{\mathrm{b}}$ Partial regression coefficient; the magnitude of the effect on DNA methylation.

${ }^{\mathrm{c}} \mathrm{CpG}$ that showed the same direction of effect in both the discovery and replication cohorts.

${ }^{\mathrm{d}}$ Genome-wide significance threshold (FDR q < 0.05).

${ }^{\text {e}}$ Successful replication defined as having the same direction of effect and a $P$-value $<0.05$ in the discovery cohort. 
Table 3. TOP5 of differentially methylated regions indicated by the bumphunting method.

\begin{tabular}{crrrcccc}
\hline Gene & Chr & Start & End & $\begin{array}{c}\text { Number } \\
\text { of Probes }\end{array}$ & Features $^{\mathrm{a}}$ & CGI $^{\mathrm{b}}$ & P-value \\
\hline $\log _{10}$ (PFOS) & & & & & & & \\
CYP2E1 & 10 & 135342560 & 135343280 & 6 & Body & island/shore & $3.75 \mathrm{E}-04$ \\
KLHL35 & 11 & 75139390 & 75139736 & 4 & Body & island/shore & $8.86 \mathrm{E}-04$ \\
SMAD & 15 & 67356310 & 67356942 & 5 & TSS1500/IGR & shore & $1.09 \mathrm{E}-03$ \\
HOOK2 & 19 & 12876846 & 12877188 & 4 & Body & island/shore & $1.65 \mathrm{E}-04$ \\
SLC17A9 & 20 & 61590751 & 61591209 & 4 & Body & island/shore & $4.83 \mathrm{E}-04$ \\
\hline \hline $\log _{10}$ (PFOA) & & & & & & & \\
GFPT2 & 5 & 179740743 & 179741120 & 4 & Body & island & $2.03 \mathrm{E}-03$ \\
ZFP57 & 6 & 29648225 & 29649084 & 21 & IGR & open sea & $1.00 \mathrm{E}-04^{\mathrm{FWER}, \mathrm{c}}$ \\
DUSP22 & 6 & 291687 & 293285 & 10 & Body & island & $7.87 \mathrm{E}-04$ \\
CYP2E1 & 10 & 135342560 & 135343280 & 6 & Body & island/shore & $3.72 \mathrm{E}-04$ \\
TCERG1L & 10 & 132910868 & 132911152 & 4 & Body & open sea & $1.52 \mathrm{E}-03$ \\
\hline Ab
\end{tabular}

Abbreviations: Chr, Chromosome; body, gene body; IGR, intergenic region; TSS, transcription start site; TSS1500, 1500 bases from TSS, FWER, family-wise error rate

${ }^{\mathrm{a}}$ Gene feature category of the methylation locus.

bRelation to CpG island.

${ }^{\mathrm{c}}$ FWER $<0.1$ 
Table 4. The average partial regression coefficient of TOP5 DMRs in the discovery and replication cohorts.

\begin{tabular}{|c|c|c|c|c|c|}
\hline \multirow{2}{*}{ Gene } & \multirow[b]{2}{*}{ Chr } & \multicolumn{2}{|c|}{ Discovery cohort } & \multicolumn{2}{|c|}{ Replication cohort } \\
\hline & & Average Coef ${ }^{\mathrm{a}}$ & Direction $^{\mathrm{b}}$ & Average Coef ${ }^{\mathrm{a}}$ & Direction $^{\mathrm{b}}$ \\
\hline \multicolumn{6}{|l|}{$\log _{10}(\mathrm{PFOS})$} \\
\hline CYP2E1 & 10 & 0.040 & + & 0.059 & + \\
\hline KLHL35 & 11 & 0.134 & + & -0.127 & - \\
\hline$S M A D$ & 15 & 0.128 & + & 0.027 & + \\
\hline HOOK2 & 19 & -0.212 & - & 0.290 & + \\
\hline SLC17A9 & 20 & 0.165 & + & 0.163 & + \\
\hline \multicolumn{6}{|l|}{$\log _{10}(\mathrm{PFOA})$} \\
\hline GFPT2 & 5 & -0.100 & - & -0.035 & - \\
\hline ZFP57 & 6 & -0.112 & - & -0.052 & - \\
\hline DUSP22 & 6 & -0.030 & - & -0.113 & - \\
\hline CYP2E1 & 10 & 0.112 & + & 0.007 & + \\
\hline TCERG1L & 10 & 0.025 & + & 0.009 & + \\
\hline
\end{tabular}

Abbreviations: Chr, Chromosome.

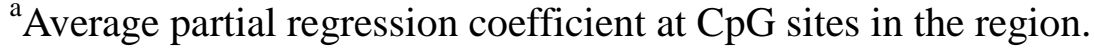

bdirection of methylation change: +, up-methylated, -, down-methylated. 

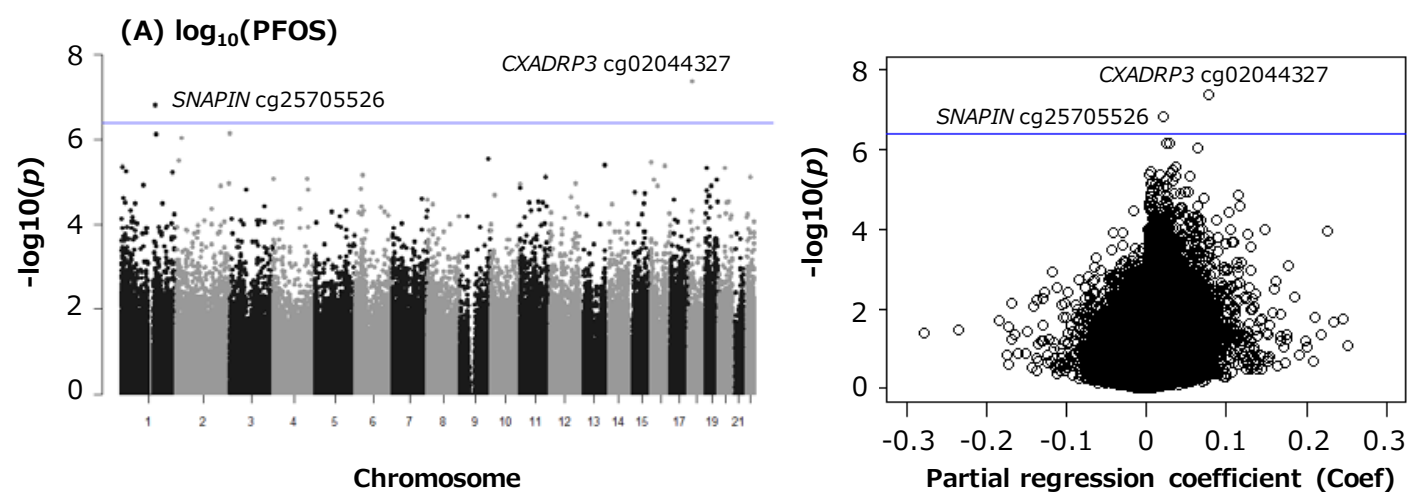

(B) $\log _{10}$ (PFOA)
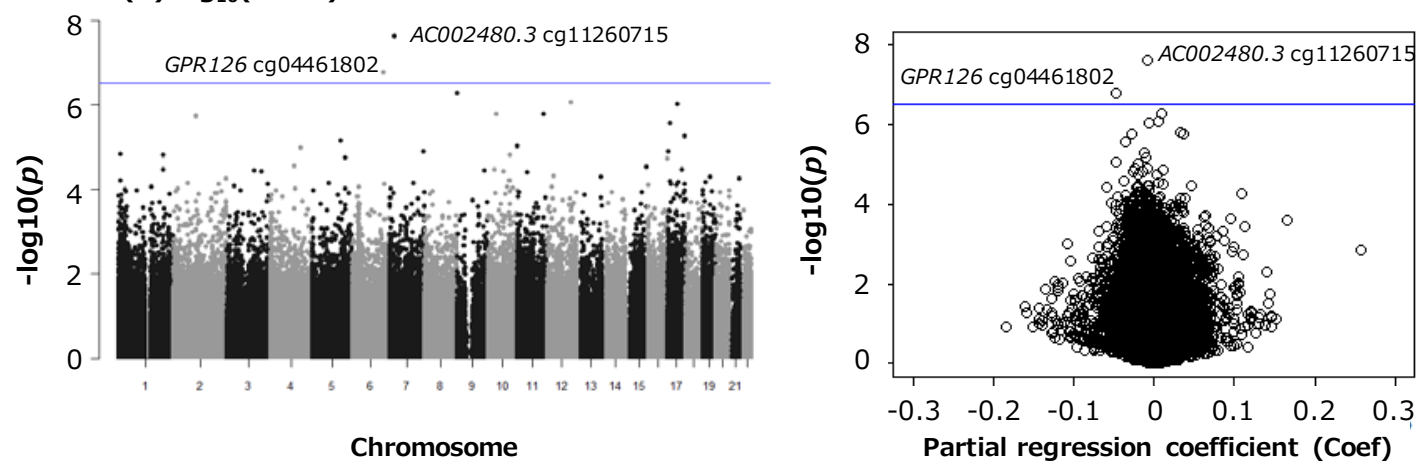

Figure 1. Manhattan (left panels) and volcano plots (right panels) of the genome-wide associations of DNA methylation with prenatal exposure to PFOS (A) or PFOA (B) in the discovery cohort.

Left panels: Manhattan plots of $P$-value for the associations between prenatal PFAS exposures and DNA methylation across chromosomes. Right panels: Volcano plots showing $P$-values versus the magnitude of effect (Coef) on DNA methylation associated with prenatal PFAS exposures. Horizontal lines represent the significance threshold of a FDR $<0.05$. 